\title{
ON THE LIMIT CYCLES SURROUNDING A DIAGONALIZABLE LINEAR NODE WITH HOMOGENEOUS NONLINEARITIES
}

\author{
JAUME LLIBRE ${ }^{1}$ AND TAYEB SALHI ${ }^{2}$
}

\begin{abstract}
In this paper we study the existence and non-existence of limit cycles for the class of polynomial differential systems of the form

$$
\dot{x}=\lambda x+P_{n}(x, y), \quad \dot{y}=\mu y+Q_{n}(x, y),
$$
\end{abstract}

where $P_{n}$ and $Q_{n}$ are homogeneous polynomials of degree $n$.

\section{INTRODUCTION AND STATEMENT OF THE MAIN RESUlts}

A polynomial differential system in $\mathbb{R}^{2}$ is a differential system of the form

$$
\frac{d x}{d t}=\dot{x}=P(x, y), \quad \frac{d y}{d t}=\dot{y}=Q(x, y),
$$

where $P(x, y)$ and $Q(x, y)$ are polynomials in the variables $x$ and $y$ with real coefficients. Then $m=\max \{\operatorname{deg} P, \operatorname{deg} Q\}$ is the degree of the polynomial system.

As usual a limit cycle of a system (1) is an isolated periodic solution in the set of all periodic solutions of system (1). Limit cycles of planar differential systems were defined by Poincaré [21] and started to be studied intensively at the end of the 1920s by van der Pol [22], Liénard [12] and Andronov [1].

In the qualitative theory of the polynomial differential equations in the plane $\mathbb{R}^{2}$ one of the more difficult problems is the study of their limit cycles. Thus the second part of the unsolved 16-th Hilbert problem [13] asked for an upper bound on the maximum number of limit cycles for the polynomial differential systems of a given degree in function of this degree, see for more details the surveys [14] and [11].

2010 Mathematics Subject Classification. Primary 34C05, 34A34.

Key words and phrases. Polynomial differential systems, polynomial vector fields, limit cycles. 
In this paper for the class of polynomial differential systems in $\mathbb{R}^{2}$ of the form

$$
\dot{x}=P_{1}(x, y)+P_{n}(x, y), \quad \dot{y}=Q_{1}(x, y)+Q_{n}(x, y),
$$

where $n>1$, and $P_{k}(x, y)$ and $Q_{k}(x, y)$ are homogeneous polynomials of degree $k$, we want to study the existence and non-existence of limit cycles.

For the polynomial differential systems (2) having a linear focus at the origin of coordinates of the form

$$
\dot{x}=\lambda x-y+P_{n}(x, y), \quad \dot{y}=x+\lambda y+Q_{n}(x, y),
$$

their limit cycles have been studied intensively, see for instance [3, 4, $5,6,8,9,10,15,17,18,20]$. But there are very few results on the limit cycles of the polynomial differential systems having a linear node at the origin of coordinates of the form

$$
\dot{x}=\lambda x+P_{n}(x, y), \quad \dot{y}=\mu y+Q_{n}(x, y),
$$

with $\lambda \mu>0$.

Recently in [2] the polynomial differential systems (3) with $\lambda=\mu$ and $n>1$ have been analyzed, proving that if $n$ is odd such systems have at most one limit cycle, and if $n$ is even then they have no limit cycles. On the other hand, in Proposition 6.3 and Remark 6.4 of the paper [7] are examples of systems (3) having two, one or zero limit cycles surrounding the origin. Finally, when $\lambda \neq \mu$ and $\lambda \mu>0$ in [16] the authors provide sufficient conditions for the non-existence of limit cycles, or for the existence of one or two limit cycles.

Using polar coordinate $x=r \cos (\theta)$ and $y=\sin \theta$ system (3) becomes

$$
\dot{r}=f_{0}(\theta) r+f(\theta) r^{n}, \quad \dot{\theta}=g_{0}(\theta)+g(\theta) r^{n-1},
$$

and in the region $R=\left\{(r, \theta): g_{0}(\theta)+g(\theta) r^{n-1}>0\right\}$ it can be studied using the differential equation

$$
\frac{d r}{d \theta}=\frac{f_{0}(\theta) r+f(\theta) r^{n}}{g_{0}(\theta)+g(\theta) r^{n-1}}
$$


where

$$
\begin{aligned}
f_{0}(\theta) & =\lambda \cos ^{2} \theta+\mu \sin ^{2} \theta, \\
g_{0}(\theta) & =(\mu-\lambda) \cos \theta \sin \theta, \\
f(\theta) & =\cos \theta P_{n}(\cos \theta, \sin \theta)+\sin \theta Q_{n}(\cos \theta, \sin \theta), \\
g(\theta) & =\cos \theta Q_{n}(\cos \theta, \sin \theta)-\sin \theta P_{n}(\cos \theta, \sin \theta), \\
P_{n}(x, y) & =\sum_{i=0}^{i=n} a_{(n-i) i} x^{n-i} y^{i}, \\
Q_{n}(x, y) & =\sum_{i=0}^{i=n} b_{(n-i) i} x^{n-i} y^{i} .
\end{aligned}
$$

Theorem 1. The polynomial differential system (3) with $n \geq 2$ has no limit cycles surrounding the origin in the region $R$ if one of the following conditions holds.

(a) $f=0$.

(b) $g=0$.

(c) $a_{0 n}=0$

(d) $b_{n 0}=0$.

(e) $g=g_{0} f / f_{0}$.

(f) $\left(n f g+(2-n) f_{0} g\right)^{2}-4 f g f_{0} g_{0} \leq 0$.

(g) $\left((2 n-1) f g_{0}-(2 n-3) f_{0} g\right)^{2}-4 f_{0} g_{0} f g \leq 0$.

The polynomial differential system (3) has at most one limit cycle surrounding the origin if the following condition holds

(h) $\left((2 n-1) f_{0} g-(2 n-3) f g_{0}\right)^{2}-4 f_{0} g_{0} f g \leq 0$.

Theorem 1 is proved in the next section.

\section{PROOFS}

For proving Theorem 1 we need the following two lemmas due to Lloyd [19].

Lemma 2. We have in a simply connected open set $V$ containing the origin the differential system in polar coordinates

$$
\dot{r}=S_{1}(r, \theta), \quad \dot{\theta}=S_{2}(r, \theta),
$$

where $S_{1}$ and $S_{2}$ are $C^{1} 2 \pi$-periodic functions such that $S_{1}(0, \theta)=0$ for all $\theta$, and $S_{2}(r, \theta)>0$ in $V$. The differential system (6) is equivalent 
to the differential equation

$$
\frac{d r}{d \theta}=\frac{S_{1}(r, \theta)}{S_{2}(r, \theta)}=S(r, \theta) .
$$

Therefore, if

$$
\frac{\partial S}{\partial r} \equiv 0, \quad \frac{\partial S}{\partial r} \leqslant 0, \text { or } \quad \frac{\partial S}{\partial r} \geqslant 0
$$

in $V$, then the differential system (6) has no limit cycles in $V$.

Lemma 3. Consider the differential system (6) defined in an annular region $A$ that encircles the origin and where $S_{2}(r, \theta)>0$. Then in $A$, the differential system (6) is equivalent to the differential equation (7). If (8) hold in A, then the differential system 6 has at most one limit cycle in $A$.

Proof statement ( $a$ ) of Theorem 1. If $f=0$ equation (5) becomes

$$
\frac{d r}{d \theta}=\frac{f_{0}(\theta) r}{g_{0}(\theta)+g(\theta) r^{n-1}} .
$$

Since $\lambda>$ and $\mu>0$ this last equation does not change sign in the region $C$. The solution $r(\theta)$ of this equation increases or decreases, so these solutions cannot be periodic in the region $R$, and consequently the polynomial differential system (3) has no limit cycles In $R$.

Proof statement (b) of Theorem 1. Since $g=0$ the differential equation (4) becomes

$$
\dot{r}=f_{0}(\theta) r+f(\theta) r^{n}, \quad \dot{\theta}=g_{0}(\theta) .
$$

The straight lines $\theta=0$ and $\theta=\pi / 2$ are invariant for system (3). So this system cannot have limit cycles surrounding the origin. This completes the proof of this statement.

Proof statement $(c)$ of Theorem 1. Since $a_{0 n}=0$ the differential system (3) has the straight line $x=0$ invariant, consequently this system has no limit cycles surrounding the origin.

The same argument used in the proof of statement (c) proves statement $(\mathrm{d})$.

Proof statement (e) of Theorem 1. Since $g=f g_{0} / f_{0}$ system (4) becomes

$$
\dot{r}=f_{0}(\theta) r+f(\theta) r^{n}, \quad \dot{\theta}=g_{0}(\theta)\left(1+\frac{f(\theta)}{f_{0}(\theta)}\right) r^{n-1} .
$$


So the proof ends following the same argument used in the proof of statement (b).

Proof statement (f) of Theorem 1. Let

$$
S(r, \theta)=\frac{f_{0} r+f r^{n}}{g_{0}+g r^{n-1}},
$$

defined in the simply connected region $R$. The derivative of $S$ with respect to $r$ is

$$
\frac{\partial S}{\partial r}=\frac{f_{0} g_{0}+\left(n f g_{0}+(2-n) f_{0} g\right) r^{n-1}+f g r^{2 n-2}}{\left(g_{0}+g r^{n-1}\right)^{2}} .
$$

Since $\left(n f g+(2-n) f_{0} g\right)^{2}-4 f g f_{0} g_{0} \leq 0$ the numerator of $\partial S / \partial r$ does not change of sign, and we can apply Lemma 2 to the differential equation (5), and the proof of this statement follows.

Proof statement $(g)$ of Theorem 1. Doing the change of variables $R=$ $\sqrt{r}$ in the region $C$, the differential equation (5) becomes

$$
\frac{d R}{d \theta}=\frac{f_{0} R+f R^{2 n-1}}{2\left(g_{0}+g R^{2 n-2}\right)}=S(R, \theta) .
$$

The derivative of $S$ with respect to $R$ is

$$
\frac{\partial S}{\partial R}=\frac{f_{0} g_{0}+\left((2 n-1) f g_{0}-(2 n-3) f_{0} g\right) R^{2 n-2}+f g R^{4 n-4}}{2\left(g_{0}+g R^{2 n-2}\right)^{2}}
$$

Since $\left((2 n-1) f g_{0}-(2 n-3) f_{0} g\right)^{2}-4 f_{0} g_{0} f g \leq 0$ the numerator of $\partial S / \partial R$ does not change of sign,, and again we can apply Lemma 2 to the differential equation (9), and statement (g) is proved.

Proof statement (h) of Theorem 1. Doing the change of variables $R=$ $1 / \sqrt{r}$ in the region $R$ the differential equation (5) becomes

$$
\frac{d R}{d \theta}=\frac{R\left(f_{0} R^{2 n-2}+f\right)}{2\left(g_{0} R^{2 n-2}+g\right)}=S(R, \theta) .
$$

So the derivative of $S$ with respect to $R$ is

$$
\frac{\partial S}{\partial R}=-\frac{f g+\left((2 n-1) f_{0} g-(2 n-3) f g_{0}\right) R^{2 n-2}+f_{0} g_{0} R^{4 n-4}}{2\left(g_{0} R^{2 n-2}+g\right)^{2}}
$$

The image of the region $R$ under the map $r \rightarrow 1 / \sqrt{r}$ is an annular region $A$, one of the boundaries of this annulus is the infinity. Since $\left((2 n-1) f_{0} g-(2 n-3) f g_{0}\right)^{2}-4 f_{0} g_{0} f g \leq 0$ the numerator of $\partial S / \partial R$ does not change of sign, we can apply Lemma 3 in the annular region $A$ to the differential equation (10), and this completes the proof of this statement. 
In short Theorem 1 is proved.

\section{ACKNOWLEDGEMENTS}

The first author is partially supported by the Ministerio de Ciencia, Innovación y Universidades, Agencia Estatal de Investigación grants MTM2016-77278-P (FEDER), the Agència de Gestió d'Ajuts Universitaris i de Recerca grant 2017SGR1617, and the H2020 European Research Council grant MSCA-RISE-2017-777911. The second author is partially supported by the Algerian Ministry of Higher Education and Scientific Research.

\section{REFERENCES}

[1] A.A. Andronov, Les cycles limites de Poincaré et la théorie des oscillations auto-entretenues, C.R. Acad. Sci. Paris 89 (1929), 559-561.

[2] A. Bendjeddou, J. Llibre And T. SAlhi, Dynamics of the polynomial differential systems with homogeneous nonlinearities and a star node, J. Differential Equations 254 (2013), 3530-3537.

[3] M. Carbonell, B. Coll and J. Llibre, Limit cycles of polynomial systems with homogeneous nonlinearities, J. Math. Anal. Appl. 142 (1989), 573-590.

[4] M. Carbonell And J. Llibre, Limit cycles of a class of polynomial systems, Proc. Royal Soc. Edinburgh 109A (1988), 187-199.

[5] C. Chicone Limit cycles of a class of polynomial vector fields in the plane, J. Differential Equations 63 (1986), 68-87.

[6] A. Gasull, J. Llibre And J. Sotomayor, Limit cycles of vector fields of the form $X(v)=A v+f(v) B v$, J. Differential Equations 67 (1987), 90-110.

[7] A. Gasull, J. Llibre And J. Sotomayor, Further considerations on the number of limit cycles of vector fields of the form $X(v)=A v+f(v) B v$, J. Differential Equations 68 (1987), 36-40.

[8] J. Giné AND J. LliBRE, Integrability and algebraic limit cycles for polynomial differential systems with homogeneous nonlinearities, J. Differential Equations 197 (2004), 147-161.

[9] C. Li, W. Li, J. Llibre And Z. Zhang, On the limit cycles of polynomial differential systems with homogeneous nonlinearities, Proc. Edinburgh Math. Soc. 43 (2000), 529-543.

[10] C. Li, W. Li, J. Llibre And Z. Zhang, New families of centers and limit cycles for polynomial differential systems with homogeneous nonlinearities, Ann. Diff. Eq. 193 (2003), 302-317.

[11] J. LI, Hilbert's 16-th problem and bifurcations of planar polynomial vector fields, Int. J. Bif. Chaos 13 (2003), 47-106.

[12] A. LiÉnARD, Etude des oscillations entretenues, Rev. Générale de l'Electricité 23 (1928), 901-912.

[13] D. Hilbert, Mathematische Probleme, Lecture, Second Internat. Congr. Math. (Paris, 1900), Nachr. Ges. Wiss. G"ttingen Math. Phys. KL. (1900), 253-297; English transl., Bull. Amer. Math. Soc. 8 (1902), 437-479. 


\section{LIMIT CYCLES OF A CLASS OF POLYNOMIAL DIFFERENTIAL SYSTEMS 7}

[14] Yu. Ilyashenko, Finiteness theorems for limit cycles, Translations of Math. Monographs 94, Amer. Math. Soc., 1991.

[15] J. Llibre AND C. VAlls, Classification of the centers, their cyclicity and isochronicity for a class of polynomial differential systems generalizing the linear systems with cubic homogeneous nonlinearities, J. Differential Equations 46 (2009), 2192-2204.

[16] J. Llibre, J. YU AND X. ZHANG, On the limit cycles of the polynomial differential systems with a linear node and homogeneous nonlinearities, Int. J. Bifurcation and Chaos 24 (2014), 1450065-pp 7.

[17] J. Llibre AND X. Zhang, On the limit cycles of linear differential systems with homogeneous nonlinearities, Canadian Math. Bull. 58 (2015), 818-823.

[18] J. Llibre AND X. ZHANG, Non-existence, existence and uniqueness of limit cycles for quadratic polynomial differential systems, Proc. of the Royal Society of Edinburgh: Section A Mathematics 149 (2019), 1-14.

[19] N.G. Lloyd, A note on the number of limit cycles in certain two-dimensional systems, J. London Math. Soc. 20 (1979), 277-286.

[20] N.G. LlOYD, Limit cycles of certain polynomial differential systems, in Nonlinear Functional Analysis and its Applications, S.P. Singh, ed NATO AS1 Series C, Vol. 173, Reidel, Dordrecht, The Netherlands, 1986, pp. 317-326.

[21] H. Poincaré, Mémoire sur les courbes définies par une équation differentielle I, II, J. Math. Pures Appl. 7 (1881), 375-422; 8 (1882), 251-296; Sur les courbes définies pas les équations differentielles III, IV, 1 (1885), 167-244; 2 (1886), 155-217.

[22] VAN DER PoL, On relaxation-oscillations, Phil. Mag. 2 (1926), 978-992.

1 Departament de Matemàtiques, Universitat Autònoma de Barcelona, 08193 Bellaterra, Barcelona, Catalonia, Spain

Email address: jllibre@mat.uab.cat

2 Department of Mathematics, University of Mohamed El Bachir El Ibrahimi Bordj Bou Arréridj, El anasser, Algeria

Email address: t.salhi@univ-bba.dz 